\title{
Late endemic syphilis: case report of bejel with gummatous laryngitis
}

\author{
J L PACE, * G W CSONKA † \\ From the ${ }^{*}$ King Abdul Aziz Military Hospital, Tabuk, Saudi Arabia, and the †Genitourinary Medicine Clinic, \\ West London Hospital, London
}

SUMMARY An elderly Bedouin woman originally thought, on clinical and histological grounds, to have tuberculosis of the larynx was found to have gummatous laryngitis due to late endemic syphilis (bejel). This disease is highly prevalent in the Bedouin tribes of the Middle East. Doctors dealing with Arab patients, either in the Middle East or elsewhere, should be aware of this possibility.

\section{Case report}

A Bedouin woman aged 72 was referred to the ear, nose, and throat clinic of the King Abdul Aziz Military Hospital, Tabuk, because of hoarseness of some three months' duration. The hoarseness had developed gradually and was accompanied by a dry cough. Swallowing and drinking were unimpaired, her general health was good and she had not lost weight. Her history and family history were non-contributory, and in particular there was no suggestion of tuberculosis.

On examination, positive clinical findings were confined to the posterior endolarynx, where a granulomatous lesion was seen at the posterior commissure. Investigations, which included routine haematology, erythrocyte sedimentation rate, and chest $x$ ray, and the tuberculin skin test and direct examination of repeated specimens of sputum for tubercle bacilli, all gave negative results. A presumptive diagnosis of laryngeal tuberculosis was made, with neoplasm and syphilis being considered as alternative diagnoses. The patient was admitted for examination and biopsy under anaesthesia. At endoscopy (performed by $\operatorname{Dr} \mathbf{R}$ Kato), hypertrophic granulomatous lesions were observed along both vocal cords. The posterior laryngeal commissure showed mucosal ulceration and erythematous changes. The epiglottis and aryepiglottic folds were normal, but the arytenoid folds were oedematous. Cord mobility was restricted because of the granulomatous changes. The pyriform sinuses and the mucosal surfaces of the lateral pos-

Address for reprints: Dr G W Csonka, Department of Genitourinary Medicine, West London Hospital, Hammersmith Road, London W6 7DQ

Accepted for publication 24 June 1987 terior wall were clear. The clinical impression was one of chronic laryngitis due to granulomatous or neoplastic disease.

Histological examination of biopsy specimens from both vocal cords showed extensive necrosis and breakdown of the covering epithelium. The deeper portions were represented by granulation tissue, which included demarcated granulomas composed of epithelioid and Langhans' giant cells with central caseous necrosis. In addition, a dense lymphocytic infiltrate was present. No tubercle bacilli were detected (fig 1). The findings were thought to be consistent with tuberculosis. In view of the repeatedly negative results from sputum, tuberculin skin test, and chest $x$ ray, this diagnosis was in some doubt. After discussion with the department of internal medicine, however, antituberculous treatment was nevertheless started. The patient was given ethambutol, isoniazid, and rifampicin, but without improvement.

At that time the Venereal Disease Research Laboratory (VDRL) test was found to give a positive result, but this was dismissed as a casual finding. Further testing, however, showed positive reactions to the Treponema pallidum haemagglutination assay (TPHA), the fluorescent treponemal antibody, absorbed (FTA-ABS) test and to the VDRL at a titre of $1 / 128$. The diagnosis was then changed to that of endemic syphilis (bejel). $X$ rays of the long bones of the patient's legs and hands showed osteoperiostitis, a probable effect of bejel on bone, which strongly supported the diagnosis. The biopsy specimen was reinvestigated in Oxford, where silver impregnation showed organisms like Treponema pallidum (fig 2 ). The patient was treated wih $2 \cdot 4$ MIU benzathine penicillin intramuscularly. A laryngeal lesion caused by bejel would be expected to clear within a few weeks after penicillin treatment. Three weeks after penicillin treat- 


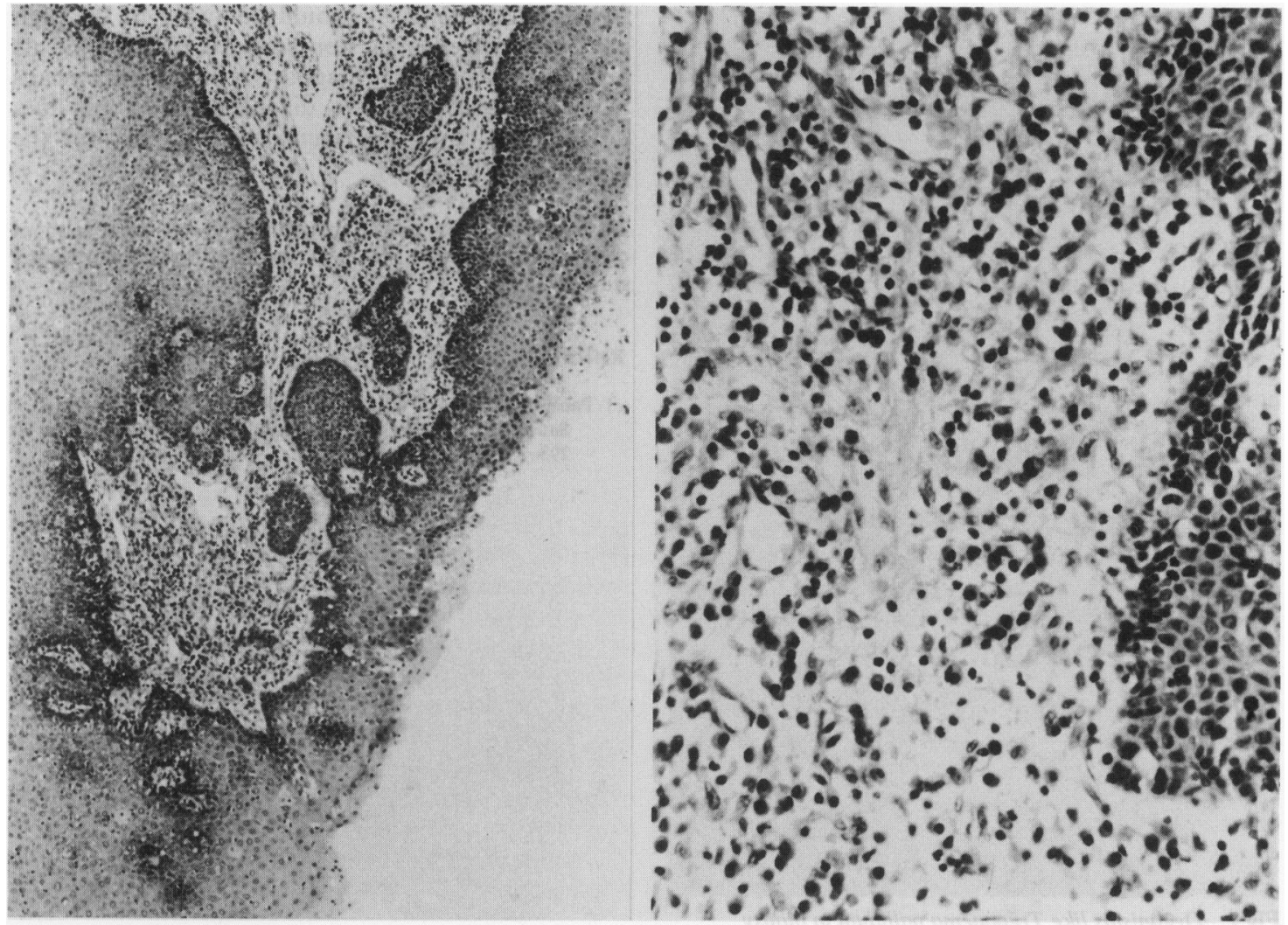

Fig 1 Section of vocal cord biopsy specimen showing necrosis, demarcated granulation tissue composed of epithelioid and Langhans' giant cells, and dense lymphocytic infiltrate. (Haematoxylin and eosin, low magnification on left, higher magnification on right.)

ment her voice had improved and the granulomatous inflammation had reduced. Improvement was maintained, and two months later the appearance of the larynx had reverted to normal and her voice was back to full resonance.

\section{Discussion}

Endemic non-venereal syphilis (bejel) is prevalent among Bedouin tribes of the Middle East, occurring in up to $27 \%$ of those born and bred in the desert. ${ }^{1}$ This disease is also found in parts of Africa and some Pacific Islands. It is contracted in early childhood, via the infectious mucous patches in the mouth, by intimate household contacts and probably by drinking from the same communal vessels as an infected person. Primary lesions are very rare, but secondary ones causing rashes are common and are usually followed by latency. If a tertiary stage develops it usually affects the skin, the long bones of the legs, and the nasopharynx. When bones and joints are affected, local pain is common, especially at night. Pronounced radiological changes showing periostitis and osteitis with cortical thickening and narrowing of the medullary space can be seen. These changes affect particularly the tibia and fibula and, less often, the radius and ulna and some other bones. When the nasopharynx is affected the patient may present with collapse of the bridge of the nose or perforation of the palate, which is associated with difficulties in speaking and eating. Occasionally there is severe destruction of much of the nasopharynx, which results in mutilating deformities necessitating plastic surgery. The larynx is commonly affected on its own, as it was in our patient, with granulomatous lesions resembling a neoplasm or granulomatous disease.

Results of serological tests for bejel do not differ from those for venereal syphilis. Unlike in tertiary venereal syphilis, cardiovascular and neurological complications have not been observed. Congenital transmission is also unknown, probably because of the length of time between contracting the infection and becoming pregnant.

Doctors who may occasionally deal with patients 


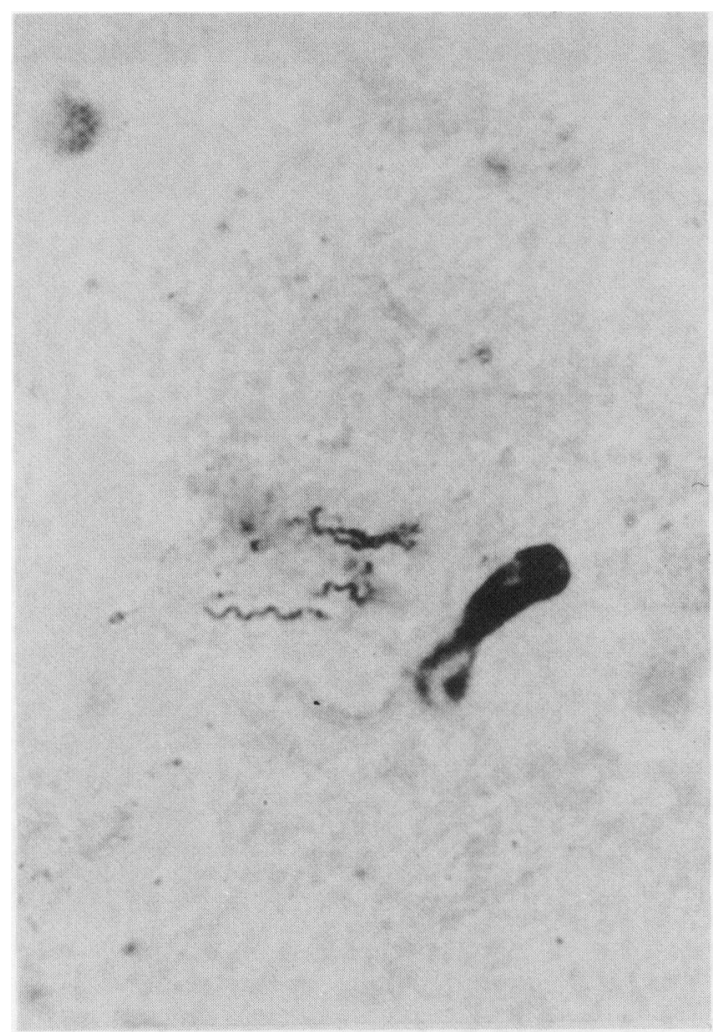

from the Middle East should be aware of the high prevalence of endemic non-venereal syphilis and its manifestations, lest the diagnosis is missed or, perhaps worse, venereal syphilis is diagnosed when this does not exist, with serious social consequences for the patient and the family.

We thank Dr V Ciucioli of the morbid anatomy department of the John Radcliffe Hospital, Oxford, for the microscopy studies.

\section{Reference}

1 Pace JL, Csonka GW. Endemic non-venereal syphilis (bejel) in Saudi Arabia. British Journal of Venereal Diseases 1984;60: 293-7.

Fig 2 Organisms like Treponema pallidum in biopsy specimen (Warthin-Starry silver impregnation). 